\title{
Erratum to: Individual and peer factors associated with ketamine use among adolescents in Taiwan
}

\author{
Kun-Hua Lee ${ }^{1} \cdot$ Yi-Chun Yeh ${ }^{2,3} \cdot$ Pin-Chen Yang ${ }^{2,3}$. \\ Huang-Chi Lin 2,3 • Peng-Wei Wang,3 - Tai-Ling Liu',3 . \\ Cheng-Fang Yen ${ }^{2,3}$
}

Published online: 8 March 2017

(C) Springer-Verlag Berlin Heidelberg 2017

\section{Erratum to: Eur Child Adolesc Psychiatry \\ DOI 10.1007/s00787-012-0292-7}

In the original publication, the scoring method of the Negative outcome expectancy for ketamine is incorrectly described. The correct version is as follows:

"The response categories included: 1, very severe damage; 2 , severe damage; 3 , a little damage; and 4, no damage."

The online version of the original article can be found under doi:10.1007/s00787-012-0292-7.

Cheng-Fang Yen

chfaye@cc.kmu.edu.tw

1 Institute of Allied Health Sciences, College of Medicine, National Cheng Kung University, Tainan, Taiwan

2 Department of Psychiatry, Kaohsiung Medical University Hospital, Kaohsiung, Taiwan

3 Department of Psychiatry, Faculty of Medicine, College of Medicine, Kaohsiung Medical University, Kaohsiung, Taiwan 